\title{
An Update of Possible Radiation-free Imaging Techniques in Dentistry
}

\author{
Maurilio D'Angelo ${ }^{1}$, Alessio Zanza ${ }^{2}$, Shilpa Bhandi ${ }^{3}$, Gabriele Miccoli $^{4}$, Andrea Cicconetti ${ }^{5}$ \\ The Journal of Contemporary Dental Practice (2021): 10.5005/jp-journals-10024-3192
}

Cone-beam computed tomography $(\mathrm{CBCT})$ represents today the most widespread and most used 3D examination in dentistry. It is precise because of its usefulness in orthodontics and orthognathic surgery with large field of view (FOVs); in oral surgery and implantology for the 3D evaluation of bone volumes and proximity to noble structures; and in endodontics for the understanding of the often difficult root canal system anatomy with reduced FOV and greater resolution that this imaging technique, also considering the wide diffusion and availability in dental offices, in addition to the short examination execution times, allows its wide use. ${ }^{1-4}$

The possibility of modifying many parameters and different aspects in this type of examination allows an easy use, speed, and optimization of radiation for diagnostic purposes, often decisive in differential diagnoses, and to facilitate surgical endodontic treatment in aid of guided or navigated surgeries. ${ }^{5-7}$

The main and most relevant dilemma in the use of this technique is represented by the presence of ionizing radiation, the importance of which is often underestimated in the prescription of this kind of diagnostic examination. ${ }^{8}$

In this regard, more and more attention is being paid to radiation-free imaging techniques, from MRI (magnetic resonance imaging), to imaging techniques that use ultrasound. The clinical dental uses of both are increasingly investigated, and the main difference is represented by the learning curve necessary to perform a good ultrasound examination, unlike MRI, which instead turns out to be a more complex examination due to the long times of acquisition of the data for having particularly high resolutions. ${ }^{9-11}$ The study of vascular lesions of the tissues of the oral cavity, of the oral tissues themselves, or of the more superficial bone and its alterations, with the appropriate probes, seems to be simple to apply, free of ionizing radiation, minimally invasive, and easily available in the office for constant use during daily clinical practice. ${ }^{10,12}$ Recent evidence makes it possible to consider MRI a complete dental diagnostic examination, which allows both to investigate the anatomy of the soft tissues, with certain frequencies, and the volumes and bone density. ${ }^{9}$ Not only that, the improvements in the evaluation of the anatomy of the endodontic system, and in the guided surgery, with templates produced exclusively on the basis of this diagnostic examination, were important. ${ }^{11}$ This would represent a major step toward radiation-free diagnostics, increasingly innovative and protective toward the patient.

Unlike resonance, ultrasound allows a more specific evaluation of a superficial mucous or bone site but allows a great possibility to modify the parameters, to increase the definition of the images collected, or the depth of vision. ${ }^{10-13}$ Moreover, specific parameters also allow the evaluation of dental hard tissues, up to now not explored with this method, noninvasive, which does not cause
1,2,4,5 Department of Oral and Maxillo-Facial Sciences, Sapienza University of Rome, Rome, Italy

${ }^{3}$ Department of Restorative Dental Sciences, Jazan University, Jazan, Kingdom of Saudi Arabia

Corresponding Author: Alessio Zanza, Department of Oral and Maxillo-Facial Sciences, Sapienza University of Rome, Rome, Italy, Phone: +39 3348731467, e-mail: ale.zanza@gmail.com

How to cite this article: D'Angelo M, Zanza A, Bhandi S, et al. An Update of Possible Radiation-free Imaging Techniques in Dentistry. J Contemp Dent Pract 2021;22(9):973-974.

Source of support: Nil

Conflict of interest: None

biological damage and is able to avoid the use of intraoral periapical or bitewing radiographs. ${ }^{11}$

Further in-depth studies and in vitro studies will be required, to ascertain the effectiveness and innovation brought about by the application of these techniques for use that is not practiced today, and subsequently, the instruments for oral use will have to be implemented, especially the probes for diagnostic examinations with ultrasound. ${ }^{10}$ The margins of success are wide, guided by the noninvasiveness of these procedures and by the absence of biological damage caused by ionizing radiation.

\section{References}

1. Perrotti G, Baccaglione G, Clauser T, et al. Total face approach (TFA) 3D cephalometry and superimposition in orthognathic surgery: evaluation of the vertical dimensions in a consecutive series. Methods Protoc 2021;4(2):36. DOI: 10.3390/mps4020036.

2. Alhammadi MS, Al-Mashraqi AA, Alnami RH, et al. Accuracy and reproducibility of facial measurements of digital photographs and wrapped cone beam computed tomography (CBCT) photographs. Diagnostics (Basel) 2021;11(5):757. DOI: 10.3390/ diagnostics11050757.

3. Perrotti G, Baccaglione G, Clauser T, et al. Total face approach (TFA): a novel 3D approach to describe the main cephalometric craniomaxillofacial parameters. Methods Protoc 2021;4(1):15. DOI: 10.3390/mps4010015.

4. Valenti-Obino F, Di Nardo D, Quero L, et al. Symmetry of root and root canal morphology of mandibular incisors: a cone-beam computed tomography study in vivo. J Clin Exp Dent 2019;11(6):e527-e533. DOI: 10.4317/jced.55629.

5. Bhandi S, Mashyakhy M, Abumelha AS, et al. Complete obturationcold lateral condensation vs. thermoplastic techniques: a systematic review of micro-CT studies. Materials (Basel) 2021;14(14):4013. DOI: 10.3390/ma14144013.

() The Author(s). 2021 Open Access This article is distributed under the terms of the Creative Commons Attribution 4.0 International License (https://creativecommons. org/licenses/by-nc/4.0/), which permits unrestricted use, distribution, and non-commercial reproduction in any medium, provided you give appropriate credit to the original author(s) and the source, provide a link to the Creative Commons license, and indicate if changes were made. The Creative Commons Public Domain Dedication waiver (http://creativecommons.org/publicdomain/zero/1.0/) applies to the data made available in this article, unless otherwise stated. 
6. Gambarini G, Ropini P, Piasecki L, et al. A preliminary assessment of a new dedicated endodontic software for use with CBCT images to evaluate the canal complexity of mandibular molars. Int Endod J 2018;51(3):259-268. DOI: 10.1111/iej.12845.

7. Gambarini G, Galli M, Stefanelli LV, et al. Endodontic microsurgery using dynamic navigation system: a case report. J Endod 2019;45(11):1397-1402.e6. DOI: 10.1016/j.joen.2019.07.010.

8. Jacobs R, Salmon B, Codari M, et al. Cone beam computed tomography in implant dentistry: recommendations for clinical use. BMC Oral Health 2018;18(1):88. DOI: 10.1186/s12903-018-0523-5.

9. Reda R, Zanza A, Mazzoni A, et al. An update of the possible applications of magnetic resonance imaging (MRI) in dentistry: a literature review. J Imaging 2021;7:75. DOI: 10.3390/jimaging7050075.
10. Reda R, Zanza A, Cicconetti A, et al. Ultrasound imaging in dentistry: a literature overview. J Imaging 2021;7:238. DOI: 10.3390/ jimaging7110238.

11. Di Nardo D, Gambarini G, Capuani S, et al. Nuclear magnetic resonance imaging in endodontics: a review. J Endod 2018;44(4):536-542. DOI: 10.1016/j.joen.2018.01.001.

12. Patil S, Alkahtani A, Bhandi S, et al. Ultrasound imaging versus radiographs in differentiating periapical lesions: a systematic review. Diagnostics (Basel) 2021;11(7):1208. DOI: 10.3390/diagnostics11071208.

13. Izzetti R, Vitali S, Aringhieri G, et al. Discovering a new anatomy: exploration of oral mucosa with ultra-high frequency ultrasound. Dentomaxillofac Radiol 2020;49(7):20190318. DOI: 10.1259/ dmfr.20190318. 